\title{
1970-2014: From Space Ion Thrusters to Nano-Tools.
}

\author{
Pierre Sudraud
}

Orsay-Physics, Tescan Orsay Holding, Fuveau, France.

In the 1970's, Albert Crewe demonstrated with scanning transmission electron microscopy that field emission sources allowed spectacular improvement (i.e., atomic resolution) in the field of electron microscopy [1]. Several groups in the USA, Great Britain, and France began to work on point ion sources in order to bring similar improvements to ion instruments, especially to secondary ion mass spectroscopy (SIMS).

Two main methods for improving ion sources began to be explored in the1970's. The first was based on the field ionization of a gas on a tip (like in Müller's field ion microscope). This principle was used successfully by Levi-Setti and Swanson's groups [2,3]. Due to technological difficulties such as poor life time, low current, the need for cryogenic temperature, etc. progress stalled during these years. The second method of research exploited the attractive electro-hydrodynamic (EHD) phenomena. At the beginning of the 1970's, great efforts were performed both in the USA and in Europe to develop ion space thrusters using this spectacular physical effect. Some physicists used EHD on liquid metal meniscus to create high brightness ion sources which were easier and cheaper to develop and industrialize than cryogenic field ion sources despite their lower brightness [4-6]. The physical study of these sources began and were renamed LMIS for liquid metal ion sources. The LMIS qualities of stability, brightness, long life time, and small size were at the origin of the focused ion beam (FIB) technology and are still appreciated by users today.

The first generation of FIB usage was often devoted to improvement of SIMS. Then, the FIB capacity for applications such as machining any solid at deep sub-micron scale, local implantation, and laser mirror polishing without any lithographic processes was exploited mainly in the 1980's at Hughes Research Labs and in Japan, especially for fully UHV optoelectronic devices processing.

However, this interest for high voltage FIB (up to $200 \mathrm{kV}$ ) declined at the end of the 1980 's and moderate voltage FIB (about $30 \mathrm{kV}$ ) showed very powerful capabilities as a support for the micro-fabrication industry. Now, FIB constitutes one of the major industrial tools for semiconductors analysis, especially for editing, failure analysis, and TEM sample preparation. The applications space is increasing like 3D mapping showing more and more promising prospects mainly in the biology sciences as shown in Figures 1 and 2.

It is amusing to see that FIB technology originated LMIS source development, and now increased demands in FIB applications are creating needs for new ion sources such as plasma sources, cryogenic point sources as gas field ion sources (GFIS) brought back by the ALIS group, ultra-cold atoms ion sources, etc. It seems that new sources will not completely replace gallium, but they will allow a larger array of FIB well adapted to many specific applications. This leads to interesting research and development efforts, both in the area of charged particles optics and in the domain of ion sources technologies [7]. My presentation will be an attempt to provide an overview on the evolution of this 
exciting area of science and technology, mainly on the new instruments and applications which have been developed during the last ten years [8].

\section{References:}

[1] AV Crewe, J Wall and J Langmore, Science 168 (1970), p. 1338.

[2] JH Orloff and LW Swanson, J. Vac. Sci. Technol. 12 (1975), p. 1209.

[3] WH Escovitz, TR Fox and R Levi-Setti, Proceedings of the National Academy of Sciences of the United States of America (1975), p. 1826.

[4] VE Krohn and GR Ringo, Appl. Phys. Lett. 27 (1975), p. 479.

[5] JF Mahoney et al, J. Appl. Phys. 40 (1969), p. 5101.

[6] P Sudraud, These d'Etat Université Paris XI Orsay, 1979.

[7] J Orloff, M Utlaut, and L Swanson in "High resolution Focused Ion Beams: FIB and Its Applications", Kluwer Academic / Plenum Publishers, New York.

[8] I thank all my colleagues from Orsay-Physics, and especially Dr. A. Delobbe and Dr. M. Talec for permanent scientific discussions and help.

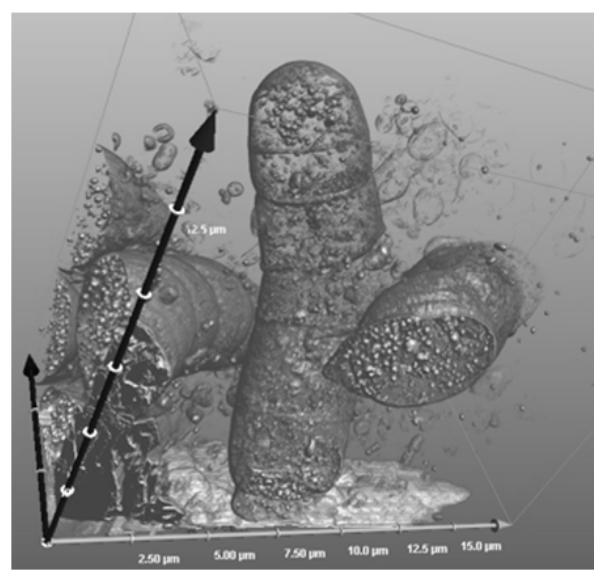

Figure 1. 3D FIB/SEM reconstruction of Algae Oscillatoria, using a LYRA3 FIB-SEM microscope from TESCAN and VSG.

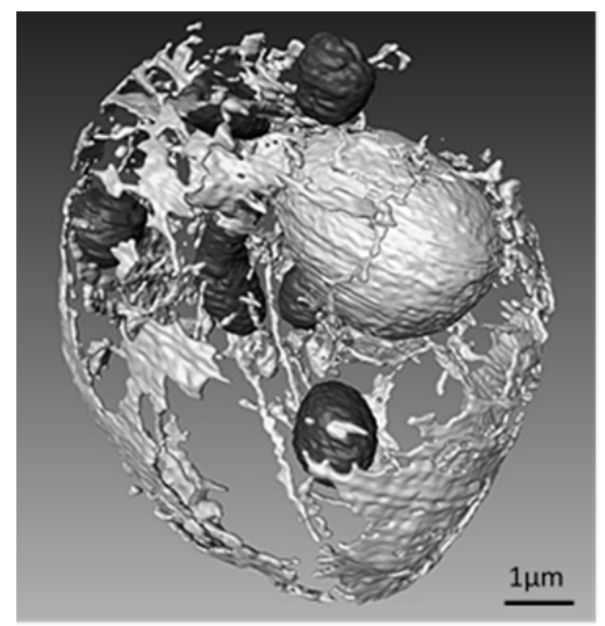

Figure 2. 3D image of a yeast cell, using a Zeiss Crossbeam microscope equipped with a COBRA FIB. 